\title{
Renate Genth/Elmar Altvater \\ Politische Konzeptionen und Schwierigkeiten der KPI in der Krise - Ein Aufriß von Problemen einer Strategie der Arbeiterbewegung (II)
}

In Prokla 26 haben wir im ersten Teil dieses Artikels die mit der ökonomischen Krise gegebene Problemlage in der italien ischen Gesellschaft analy sier $t$ und zu zeigen versucht, daß die Krise von der bürgerlichen Klasse als politisches Disziplinierungsmittel gegen die Arbeiterklasse gewendet wird. Weiter haben wir versucht, die Tradition der heute wirksamen Strategievorstellungen der KPI darzustellen, um damit zu verdeutlichen, daß die Konzeptionen einer Politik in der Krise nicht nur ad hoc-Antworten sind, sondern ihre Wurzeln in der Geschichte, in den Erfahrungen der italienischen Arbeiterbewegung haben. Im Folgenden werden wir auf einige der konkreten politischen Vorstellungen zur Überwindung der Krise eingehen und zu zeigen versuchen, welchen Schwierigkeiten die italienische Arbeiterbewegung dabei ausgesetzt ist. Von der Art und Weise der Bewältigung dieser Schwierigkeiten hängt es wesentlich $\mathrm{ab}$, ob die Ziele der Strategie - nämlich den Angriffen auf das materielle Lebensniveau und auf die politischen Errungenschaften entgegenzutreten und gleichzeitig die Hegemonie der Arbeiterklasse in der Gesellschaft auszubauen - erreicht werden können oder nicht. Die Grundfrage lautet: „Wie findet man aus der gegenwärtigen Krise einen Ausweg mit der Perspektive, auf dem Wege zum Sozialismus voranzukommen?" (1) Wie schwer die Antwort ist, verdeutlicht gerade die Situation der italienischen Arbeiterbewegung im Frühjahr 1977 (2), in einer dramatisch zugespitzten Lage wie kaum jemals zuvor nach dem zweiten Weltkrieg.

\section{Die Gefahr der Zersplitterung der Linken in der Krise}

Nicht nur die Angriffe des Kapitals und seiner politischen Kräfte in den Parlamenten, Verbänden, Regierungen sind für die Arbeiterbewegung eine Gefahr, sondern mehr noch die Tendenzen der Zersplitterung, des Bruchs zwischen Gewerkschaften und Parteien, zwischen den vom Kapital ausgebeuteten, beleidigten, marginalisierten Schichten und den politischen Organisationen der Arbeiterbewegung. Nach dem großen Wahlsieg der KPI vom Juni 1976 hat sich in der sozialistischen Partei (PSI), in der KPI und in der neuen Linken ein Prozeß des Auseinanderfallens zugetragen, der „uns Angst macht" - wie Rossana Rossanda schreibt (3).

- Die Gruppen von Lotta Continua haben sich bis auf wenige Reste selbst aufgelöst; es existiert gegenwärtig beinahe nur noch die Zeitung der Gruppe.

- Die „Partei der proletarischen Einheit für den Kommunismus“ (PdUP) hat sich in zwei Richtungen gespalten, ebenso wie „Avantguardia Operaia“. Eine Neuformierung der Linken jen-

1 Eric Hobsbawm/Giorgio Napolitano, Auf dem Weg zum ,historischen Kompromißs. Ein Gespräch über Entwicklung und Programmatik der KPI, Frankfurt/Main 1977, S. 83

2 Dieser zweite Teil des Artikels wurde Mitte April abgeschlosssen. Angesichts der gegenwärtig ungemein zugespitzten politischen Situation in Italien kann diese hier vorgelegte Einschätzung nur vorläufigen Charakter haben, sofern nicht nur Strukturprobleme herausgearbeitet sondern auch Interpretationen jüngster Entwicklungen versucht werden.

Rossana Rossanda in Il Manifesto, 8.4.1977 
seits der KPI macht mehr Schwierigkeiten als je zuvor, zumal die Gewerkschaftsflügel der gespaltenen Parteien die Spaitung mit der einen oder anderen Fraktion nicht mitgemacht haben. Mit der immer bedrohlicher werdenden Arbeitslosigkeit haben sich neue , autonome Gruppen" gebildet, die infolge der mit Dauerarbeitslosigkeit einhergehenden Perspektivlosigkeit ihrer gesellschaftlichen Existenz, anders als die autonomen Gruppen aus den Klassenkämpfen 1969, zu einem Sprengsatz innerhalb der Linken, gegen die linken Organisationen und insbesondere gegen die KPI werden können, zumal sich unter die ,Autonomen“ auch faschistische Kräfte gemischt haben.

Die KPI hat sich in einel Reihe von Ereignissen, so während der Besetzung der Universität Rom im Februar 1977 und teilweise auch gegenüber den Gewerkschaften und den Forderungen der Fabrikräte, oder bei den großen Demonstrationen in Bologna nach der Ermordung eines Studenten durch die Polizei als ,staatstragende Kraft" verhalten - sicherlich mit der Zieisetzung, den gesellschaftlichen Zersetzungsprozeß aufzuhalten, um den damit kalkulierenden Kräften entgegenzuarbeiten, aber doch mit dem Effekt, daß sich nun die Zerrissenheit in der KPI selbst zeigt und Teile der KPI und der Gewerkschaften miteinander in Konflikt geraten sind.

In einer solchen Situation tut die Rechte alles, um dieses Auseinanderfallen noch zu schüren: durch notorische Ablehnung aller Bündnisangebote zur Bewältigung der Krise, durch ein bewußtes Sich-Ausspielenlassen der Krisenmechanismen und selbst durch Provokationen wie die Ermordung des Studenten in Bologna (dem roten Beispiel einer fortschrittlichen KPI-Kommunalpolitik) oder Terroranschläge und Entführungen. die an die Zeit der Strategie der Spannung Ende der 60er/Anfang der 70er Jahre anknüpfen (4).

Aus diesen wenigen Andeutungen wird klar, daß die zugespitzte ökonomische Krise des Kapitals auch eine politische Krise der italienischen Linken hervorgerufen hat. Daher ist es nicht nur entscheidend, daß die Angriffe des Kapitals und der Regierung auf den verschiedenen Ebenen zurückgeschlagen werden, sondern daß Anstrengungen unternommen werden, den Zerfallsprozeß der sozialen Basis der Linken und die damit verbundene Zersplitterung der politischen und gewerkschaftlichen Organisationen der Linken aufzuhalten. Wenn dies nicht gelingt, dann ist die Niederlage der italienischen Klassenbewegung nur eine Frage der Zeit, trotz der immensen Erfolge der italienischen Linken während der letzten Jahre (5).

4 Mit der "Strategie der Spannung“ versuchten rechte Kräfte, insbesondere die Neofaschisten, die Linke in Mißkredit zu bringen, indem sie mit Attentaten und Terroraktionen den Ruf nach ,law and order" zu provozieren trachteten. Die einheitliche Aktion der gesamten Linken gegen diese faschistischen Provokationen brachte die Strategie der Spannung zu Fall.

5 Ein Indiz für die Gefahr von Niederlagen ist das Ergebnis bei den Kommunalwahien in einigen Städten im April 1977. Während in Rovigo (Venetien) die KPI ihre Position ausbauen konnte, mußte sie in Castellamare di Stabia (praktisch ein Vorort von Neapel) eine schwere Niederlage einstecken. Dieses Ergebris ist um so bemerkenswerter als Neapel und die anderen Städ te am Golf das Musterbeispiel für christdemokratische Korruption und Verwaltungsunfähigkeit sind. Hier eine Niederlage einstecken zu müssen, bedeutet: Die KPI hat mit ihrem Reformprogramm große Hoffnungen geweckt, ohne sie aber schnell genug erfüllen zu können. Im Prozeß der Desiliusionierung hinsichtlich möglicher Alternativen wenden sich die Wähler von der KPI ab. Es muß betont werden, daß diese Interpretation vorläufig und einseitig ist: Es soll nur eine gefährliche Tendenz für die italienische Arbeiterbewegung benannt werden. 


\section{Die Widersprüche innerhalb der KPI}

Die in der Krise besonders zugespitzten gesellschaftlichen Widersprüche durchziehen auch die kommunistische Partei, die dafür eine Lösung finden muß, um nicht politisch geschwächt zu werden. Durch die großen Erfolge in den vergangenen Jahren war es möglich, daß die KPI sich im politischen Institutionensystem fest verankert hat: in den Gemeinden, in den Regionen und nach den Wahlen vom Juni 76 verstärkt auch auf zentralstaatlicher Ebene. Daraus ist der KPI aber eine besondere Problematik erwachsen, die darin besteht, daß sie als eine Art heimliche ,Regierungspartei" $\mathrm{mit}$ ihrem Charakter als „Kampfpartei“" in Konflikt gerät.

Da die regierende Christdemokratie aufgrund der parlamentarischen Mach tverhältnisse nur auf eine Minderheit von Abgeordneten $(38,7 \mathrm{vH})$ zurückgreifen kann, ist sie auf parlamentarische Unterstuitzung der anderen Parteien angewiesen. Eine Koalitionsregierung des „nationalen Notstands" wie sie die KPI als Schlußfolgerung aus ihrem Konzept des „historischen Kompromisses“ und aus ihrer Einschätzung der ökonomischen und gesellschaftlichen Krise immer wieder vorschlägt, ist von den Christdemokraten bisher notorisch abgelehnt worden. Eine alternative Linksregierung könnte sich aufgrund der Stimmergebnisse noch nicht einmal auf $50 \mathrm{vH}$ stützen (6), so daß auf parlamentarischer Ebene eine Paitsituation entstanden ist. Sie wird gegenwärtig durch eine Politik des ,Nicht-Mißtrauens" seitens der Sozialdemokraten, Republikaner, Sozialisten und der KPI überbrückt: Regierungsvorlagen finden zwar keine zustimmende Mehrheit, aber auch infolge der Stimmenthaltung der KPI-Abgeordneten (und derjenigen anderer Parteien) keine ablehnende Mehrheit, die zum Sturz der Regierung führen würde. So kann die Minderheitsregierung Andreotti bei regelmäßiger Konsultation der „Parteien der Stimmenthaltung“ regieren. De facto regiert also die KPI mit, ohne jedoch formell Regierungsverantwortung zu tragen und ihre Vorstellungen in die Formulierung eines Regierungsprogramms einbringen und politisch durchsetzen zu können. Die Politik der Stimmenthaltung gerät in die Gefahr, dadurch die Attacken der Regierung Andreotti gegen die Interessen der Arbeiterklasse de facto mitzutragen, daß die Regierung durch das Nicht-Mißtrauen gehalten wird (7). Die KPI gerät so mit der eigenen Klassenbasis, mit anderen linken Gruppen und vor allem mit den Gewerkschaften in Konflikt. Das Dilemma als ,Regierungspartei" die Politik Andreottis passiv zu tragen, und als Kampfpartei eben diese Politik zu bekämpfen, kann sich zu einem schweren Konflikt innerhalb der Partei ausweiten.

Wir haben im ersten Teil des Aufsatzes angedeutet, daß die KPI diesen Widerspruch durch eine politische Reformprogrammatik zu lösen versucht. Durch Reformen soll das ,alte Akkumulationsmodell" verändert, die gesellschaftliche Machtposition der Arbeiterklasse durch Reform- und Demokratisierungsstrategien ausgeweitet werden. Diese Strategie wird gleichzeitig als Schritt auf dem Weg zum Sozialis-

6 Bei den Wahlen zum Parlament (Senat) erhielten am 20. Juni 1976 die KPI 34,4 vH $(33,8)$, die DC 38,7 vH $(38,9)$, die PSI 9,6 vH $(10,2)$, die Democrazia Proletaria 1,5 vH $(0,2)$, die PSDI 3,4 vH $(3,1)$.

7 Diese Tatsache wird in dem Spottnamen Regierung ,Berlingotti“ (Zusammenziehung aus Berlinguer und Andreotti) auszudrücken versucht. 
mus verstanden (98). Diese Reformstrategie hat aber im KPI-Verständnis noch eine weitere Seite: Mit ihr versucht die KPI, den in der Krise bedrohlichen korporativen Zerfall der Gesellschaft aufzuhalten, indem strukturelle Veränderungen angestrebt werden, die die materielle Basis für die Fülle von (korporativen) Sonderinteressen und ihre Durchsetzungsversuche, also für die „Disgregation" (9) auch auf Seiten der Arbeiterklasse und der anderen ausgebeuteten Schichten beseitigen soll. Das Problem allerdings besteht darin, daß gesellschaftliche Reformen als Schritt auf dem Weg zum Sozialismus so lange abstrakt sind, wie tatsächlich in der Gesellschaft die durch die Krise gegeneinander getriebenen Interessen bestehen: solange beispielsweise Kurzarbeiter in der Industrie aus der "cassa d'Integrazione" $(10)$ einen beinahe 100prozentigen Lohnausgleich erhalten, während Hunderttausend von Arbeitslosen unter dem Existenzminimum leben müssen; solange enorme Lohnunterschiede zwischen Gruppen im öffentlichen Dienst und den ausgepowerten Beschäftigten in kleinen Klitschen auf dem Lande bestehen; solange städtisches und ländliches Elend bei gleichzeitig protzig zur Schau gestelltem Reichtum der Spekulanten existieren; solange den Massen der akademischen und nicht-akademischen Jugend keine Beschäftigungsmöglichkeit, keine sinnhafte Perspektive geboten werden kann.

Die KPI hat sich mit der Reformprogrammatik als Protagonist gegen die aus diesen Widersprüchen resultierende gesellschaftliche Disgregation stark exponiert, ohne allerdings ökonomisch und politisch in der Lage zu sein, die materiellen Gründe für die korporativen Gegensätze kurzfristig aufzuheben oder doch einzuschränken. Unmittelbare Verteidigung der Lebensinteressen und Einsatz für gesellschaftliche Reformen fallen nicht zusammen und daher ist es in der Krise besonders schwer, eine Politik zu formulieren und durchzusetzen, die vereinheitlichend wirkt. Mit ihrer Reformprogrammatik hat die KPI bislang diese Vereinheitlichung eher appellhaft betrieben und infolgedessen haben sich Kämpfe, Organisationsformen, neue Gruppen entwickelt, die jenseits und zum Teil gegen die KPI ihre Interessen durchzusetzen versuchen. Als heimliche „Regierungspartei“" hat die KPI ihnen gegenüber an

8 So meint Giorgio Napolitano: ,Die sozialen Zielsetzungen der neuen Entwicklungspolitik, die wir als Ausweg aus der Krise vorschlagen, sind die der größtmöglichen Beschäftigung, der Emanzipation der Bevölkerung der süditalienischen Regionen von Rückständigkeit und Elend und der Übergang der gesamten Nation zu einer besseren, kulturell und sozial fortschrittlicheren Lebensweise, die nicht länger verzerrt und entfremdet wäre. Hat all dies nichts mit Sozialismus zu tun, mit dem Ziel einer sozialistischen Transformation der Gesellschaft?* (Hobsbawm/Napolitano, a.a.O., S. 88)

9 „,disgregazione“ bezeichnet den Zerfalls- und Zersetzungsprozeß gesellschaftlicher Strukturen, die naturwüchsige Auseinander- und Gegeneinanderentwicklung von gesellschaftlichen Interessen und Gruppen,die auch gemeinsame Zielvorstellungen haben können. Es geht dabei also nicht um die Gegensätzlichkeit des Klassenkonflikts, sondern um ihn überlagernde Konflitkebenen, die sich selbst durch die Arbeiterklasse aufgrund der in der Krise besonders forcierten Konkurrenz hindurchziehen können, wenn dem nicht bewu\}t politisch entgegengearbeitet wird.

10 Mit der cassa d'integrazione ist es 1974 den Gewerkschaften gelungen, für Industriearbejter einen Ausgleichsfonds für Kurzarbeit einzurichten, aus dem den Kurzarbeitern last der volle Bruttolohn weitergezahlt wird - eine der wichtigsten Errungenschaften der it:alienischen Gewerkschatsbewegung in den letzten Jahren. 
Glaubwürdigkeit verloren, die nur zurückzugewinnen ist, wenn die angestrebten und versprochenen Reformen nicht nur auf dem Papier bleiben. In der Krise können Reformen aber nur kämpferisch durchgesetzt werden. Das heißt aber: Die Alternative „Regierungspartei“" oder „Kampfpartei“ ist nicht zu parallelisieren mit der Alternative reformistische Partei oder revolutionäre Partei. Die Integrationsfähigkeit der Partei, d. h. ihre Erfolgsaussichten bei der Abwehr der Disgregation auch innerhalb der ausgebeuteten Klassen, hängen von Reformmaßnahmen ab, die aber nur kämpferisch gegen die Regierung durchgesetzt werden können.

Es kann keineswegs gesagt werden, daß das autonome Kampfpotential, das sich scheinbar links von der KPI heranbildet, durchgängig fortschrittlich sei, weil die Auseinandersetzungen sehr militant geführt werden (11). In ihrer korporativen Disparität richten sich die Interessen der in den ,Autonomen“ zusammengefaßten Gruppen gegen die auf die Gesellschaft insgesamt bezogenen Perspektiven der Arbeiterklasse, der Gewerkschaften und der KPI. Antigewerkschaftliche Tendenzen haben in den jüngsten Auseinandersetzungen ein starkes Gewicht gehabt, ebenso wie eine bei vielen kompromißlose Ablehnung der KPI-Politik, die die Basis für einen neuen Antikommunismus werden kann. Aber andererseits kehrt sich die Notwendigkeit heraus, daß Gewerkschaften und die KPI als größte, ,hegemoniale" Organisationen der italienischen Arbeiterklasse die in den Revolten zum Ausdruck kommenden Interessen positiv aufgreifen, und nicht durch moralische Levitenlese oder Repression auf die falsche Seite, die Instrumentalisierung dieser autonomen Bewegungen durch die autoritäre Rechte, treiben (12).

11 Es sind insbesondere während der Studentenkämpfe der letzten Monate neue Gruppen hervorgetreten, wie ,autonomia operaia" oder die ,,ind iani metropolitani“" (Stadtindianer), die jenseits der traditionellen Organisationen der Linken und zum Teil gegen sie ihre Aktionen durchführen. Von ihnen wird nicht nur die KPI heftig kritisiert und angegriffen, auch Manifesto und andere linke Organisationen sind Gegenstand ihrer Kritik. Die Gruppen sind nicht fest organisiert - was allein deshalb nicht geht, weil ihre Mitglieder sich zum allergrößten Teil aus Arbeitsiosen rekrutieren und infolgedessen außerhalb des Produktionsprozesses und ohne gewerkschaftliche Bindungen eher auf situationistischer Basis zusammenfinden. So kommt es auch, daß die Militanz in den Auseinandersetzungen weitgehend perspektivlos bleibt, allenfalls vermitteit über eine libertär interpretierte marxistische Theorie von Marcuse, Mattick, Sohn-Rethel, Agnes Heller bis zu Toni Negri. Das Problem besteht darin, daß sich in die ,autonomen" Gruppen offenbar mit Leichtigkeit auch faschistische Elemente haben mischen können, die als agents provocateurs die Verschärfung der law-and-order-Tendenzen betreiben.

12 Giorgio Amendola, den man mit Einschränkungen als Exponent des ,rechten "Flügels der KPI bezeichnen kann, hat sich sehr scharf gegen die neue Studentenbewegung ausgesprochen, ebenso wie er die gewerkschaftlichen Versuche zur Sicherung der Reallöhne und deren Ablehnung der Austeritätspolitik scharf kritisierte. Für Amendola ist ,,der Extremismus der schlimmste Feind der Arbeiterbewegung". In der neuen Studentenbewegung sieht er dementsprechend ein "Anzeichen der Wiederkunft des Faschismus". Die Gewerkschaften mit ihren Maßnahmen zur Verteidigung der Arbeiterinteressen klagt er eines „Arbeiterkorporatismus" an. Man muß allerdings betonen, daß diese Position, die er vor dem Zentralkomitee der KPI am 14.3.1977 vortrug, sehr heftig von der Mehrheit kritisiert worden ist. Vgl. den Bericht in Politique Hebdo, Nr. 263, 3.4.1977 


\subsection{Die Konflikte um die wirtschaftspolitische Konzeption der KPI}

Wie schwer sich die KPI tut, die Strategie der Reformen mit konkreten „Rezepten“ gegen die Krise zu verbinden, zeigt sich auch an den wirtschaftspolitischen Konzeptionen, auf die wir jetzt kurz eingehen wollen. Im Wahlprogramm 1976 stellte die KPI Forderungen zur Behebung der strukturellen und konjunkturellen Krise auf: es müsse die Inflation eingedämmt, das Loch in der Zahlungsbilanz gestopft, das Staatsdefizit verringert, die Arbeitslosigkeit beseitigt werden. Langfristig komme es darauf an, das Ungleichgewicht zwischen Nord und Süd aufzuheben, die Produktivität der Industrie zu steigern, die Landwirtschaft zu modernisieren, Arbeitsplätze für einige hunderttausend Jugendliche zu schaffen, den sozialen Konsum (Schulen, Krankenhäuser, öffentliche Verkehrsmittel, Wissenschaft und Kultur usw.) zu fördern, mit einer Staatsreform den Verwaltungsapparat zu entbürokratisieren, den Sektor der Staatsindustrie zu entfilzen. Gleichzeitig wird versichert, daß man „das breite Potential unternehmerischer Fähigkeiten besser für die ökonomische Entwicklung nutzen“ wolle, daß ein ,neuer Konsens zwischen Plan und Markt" herbeigeführt werden solle mit der öffentlichen Nachfrage als ,Bezugspunkt" (13).

Für das ökonomische Forschungsinstitut der KPI (Cespe) sind eine Erhöhung der Investitionsquote und staatliche Orientierung (,capitalismo orientato") die Eckwerte eines Übergangsprogramms. Gleichzeitig werden Maßnahmen entwickelt, mit denen die hohen Arbeitskosten gesenkt werden sollen (Vgl. dazu Teil I). Das Cespe verspricht sich davon eine Senkung der Inflationsrate. Darauf ist überhaupt die wirtschaftspolitische Konzeption zentriert, denn die Inflation wird vom Cespe zum „Hauptfeind“ erklärt. Inflation bedeute gesellschaftliche Zersetzung, führt insbesondere Amendola aus (14), daher die Gefahr eines autoritären Regimes wie die Beispiele aus den Inflationen der 20er Jahre oder aus Lateinamerika zeigen. Infolgedessen unterstuitzen die Vertreter dieser These auch ein rigides Sparprogramm. Amendola spricht von der Notwendigkeit, daß die Arbeiterklasse in der Krise „Opfer" bringen muß, um den Zerfall der Gesellschaft in sich bekämpfende und die Struktur der Gesellschaft zersetzende Gruppeninteressen zu verhindern. Die Arbeiterklasse solle eine ,höhere öffentliche Moral“, eine größere „,nationale Disziplin" aufbringen, das Austerity-Programm also akzeptieren. Gegenleistungen für die zu bringenden Opfer können nach Amendola nicht in irgendwelchen materiellen Erfolgen bestehen, sondern darin, daß das Land insgesamt die Krise zu überwinden vermag. Hegemonie der Arbeiterklasse setzt sich nach dieser - idealistischen - Vorstellung über beispielhafte Moral durch, bei Absehung von materiellen Interessen einerseits und von der Gegensätzlichkeit von Interessen und Moral in der Klassengesellschaft andererseits (15).

13 La Proposta Comunista, herausgegeben von der KPI zu den Juniwahlen.

14 Giorgio Amendola, in: Corriere della Sera, 9.1.1977; ders., Quel che bisogna colpire quel che bisogna attuare, in: La Proposta Comunista; ebenso viele andere Vertreter der KPI vor allem aus dem ökonomischen Forschungsinstitut (Cespe) der KPI.

15 Man darf allerdings nicht ïbersehen, daß der Kern von Amendolas auf das "Gemeinwoh "* bezogener Moral antifaschistisch ist. Vgl. Giorgio Amendola, Intervista sull:antifschismo, a cura di Piero Melograni, Roma ınd Bari 1976 
Die Konzentrierung der politischen Perspektive zur Krisenüberwindung auf antiinflationistische Maßnahmen gibt notgedrungen den Zusammenhang zwischen dem Kampf für die Verbesserung oder doch zumindest die Erhaltung der Arbeitsund Lebensbedingungen innerhalb des kapitalistischen Systems und der mittelfristigen Perspektive der sozialistischen Umwälzung auf und beschränkt sich ganz auf die Aussicht der Wiederherstellung der in der Krise aus den Fugen geratenen „,normalen“" kapitalistischen Reproduktionsbedingungen. Der Vorwurf an die diesen politischen Vorschlägen zugrundeliegenden Analyse der gegenwärtigen Krise, sie bewege sich ganz in Kategorien der bürgerlichen Ökonomie und könne daher auch keine wirklichen politischen Aiternativen erarbeiten, ist zweifellos berechtigt -- auch wenn vom Cespe bestritten wird, daß die vorgeschlagene Senkung der Arbeitskosten die Reallöhne der Arbeiterklasse angreifen müsse. Die Eindämmung der Inflation solle vielmehr durch Produktivitätssteigerungen erfolgen. Aber auch mit einer solchen wirtschaftspolitischen Therapie kann sich das Cespe (und damit die KPI) nicht der Widersprïchlichkeit der Krise entziehen: Sollten Produktivitätssteigerungen wirklich gelingen und die Arbeitskosten senken, dann kann zwar unter Umständen die Inflationsrate gesenkt werden, aber die damit einhergehenden Rationalisierungen werden die Arbeitslosigkeit eher anschwellen lassen als sie eindämmen. Hier stellt sich das in der italienischen Arbeiterbewegung breit diskutierte Problem der „compatibilita", der Vereinbarkeit von wirtschaftspolitischen Maßnahmen im Interesse der Arbeiterklasse mit dem System. Die Vorschläge des Cespe sind zum großen Teil mit dem System vereinbar, aber ob sie im Interesse der Arbeiterklasse liegen, ist mehr als fragwürdig (16).

Hier liegt die eigentliche Konfliktlinie zwischen Vertretern der KPI, die der Arbeiterklasse eine protagonistische Funktion im Sinne einer moralischen Führungsrolle beimessen, und den Gewerkschaften und großen Teilen der KPI selbst, die sich für die Verteidigung der Lebensinteressen und vor allem für die Beseitigung der Arbeitslosigkeit als „Hauptfeind“ einsetzen. Die Auseinandersetzung geht also um unterschiedliche politische und ökonomische Prioritäten, die ihre Entsprechung in unterschiedlichen Einschätzungen des Charakters der Krise, der politischen Maßnahmen $\mathrm{zu}$ ihrer Überwindung und in unterschiedlichen Verhaltensweisen zu den soziaien Konflikten und den daraus entstandenen, ,autonomen" Bewegungen haben. Diese Auseinandersetzungen reproduzieren sich bis in die höchsten Entscheidungsgremien der KPI hinein. Im Oktober 1976 und dann wieder im März 1977 haben sich schwere Konflikte $z$ wischen verschiedenen Exponenten von, Flügeln" der Partei herausgebildet, die schwersten Konflikte seit der Krise um den Austritt bzw. Ausschluß der Manifesto-Gruppe 1969. Zwar ist die Einheitlichkeit der Partei dadurch keineswegs bedroht; den Führungsgremien und insbesondere dem Vorsitzenden Berlinguer ist es bislang immer noch erfolgreich gelungen, $z$ wischen den unterschiedlichen, ja gegensätzlichen Positionen zu vermitteln. Aber der politische Sprengstoff in den unterschiedlichen Einschätzungen der Krise und der politischen Prioritäten bei ihrer Be-

36 Vg. dise Dikussion in Rinascta zrischen dem Cespe und Claudio Napoleoni auf der ei-

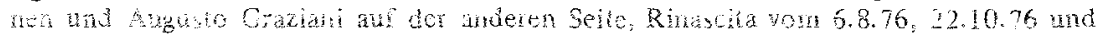
1211.76 
kämpfung ist keineswegs entschärft und könnte sich bei einer weiteren Zuspitzung der sozialen Konflikte erneut entzünden (17).

\subsection{Vorstellungen zur Staatsreform (18)}

Die Staatsreform stellt einen weiteren wichtigen Komplex des Problemwusts dar, den die Arbeiterbewegung, will sie strategisch fortschreiten, lösen muß. Denn sie hat die Erfahrung gemacht, daß viele erkämpfte Resultate, die in Form von Gesetzen oder auch Tarifverträgen bereits verfügbar sind, Erfolge auf dem Papier bleiben, wenn die staatliche Aktion nicht demokratisch kontrolliert wird.

Der Komplex der Staatsreform umfaßt einen außerordentlich vielfältigen Bereich und kann hier nur ansatzweise umrissen werden. Der öffentliche Sektor in Italien ist durch eine extreme Zentralisierung gekennzeichnet. Wichtige Bereiche wie das finanzielle Verteilungssystem, Schule, Polizei etc. stehen weitgehend nicht unter regionaler oder kommunaler Verwaltung, sondern sind organisatorisch in Rom zentralisiert. Die Gemeinden und Regionen werden zwar autonom verwaltet, sie sind aber finanziell von der römischen Zentralverwaltung abhängig. Durch eine Steuerreform in den vergangenen Jahren ist die Zentralisierung noch verstärkt worden. Gemeinden und Regionen können keine Steuern selbständig erheben; die Steuerabgaben gehen direkt nach Rom und nehmen ihren Rückweg in die Gemeinden und Regionen nur über die römische Zentrale. Angesichts der Politik der Pfründevergabe, durch die die DC in den vergangenen Jahrzehnten den gesamten Verwaltungsapparat mit ihrer Klientele durchdrungen hat, stellt die Zentralisierung eine erhebliche Machtbedingung der DC dar, macht sie beinahe identisch mit dem Staatsapparat und bietet die Grundlage zur Erhaltung ihrer Stärke trotz der Wahlresultate, die zu einer Stärkung der Linken geführt haben. Gesetze, die auf Druck und mit Beteligung der Arbeiterbewegung zustande gekommen sind, können von der DC-Klientele unterlaufen werden, wenn sie sich gegen diese richten. Zum Beispiel werden notwendige finanzielle Mittel nicht genehmigt oder kommen, wie im Fall der Sanierung Venedigs gar nicht an ihrem Bestimmungsort an; wohin sie verschwunden sind, ist unaufspürbar (20).

17 Bei den Kontroversen im ZK geht es im wesentlichen um die Frage, ob die Arbeiterklasse in der Krise Opfer ohne materielle und politische Gegenleistungen bringen muß3. Wird nämlich die - vor allem von Amendola vertretene - These von der Notwendigkeit der Opfer akzeptiert, dann bedeutet das auch die Akzeptierung des Austerity programms und die Ablehnung aller in der Klassengesellschaft notwendig partiellen Kämpfe der Arbeiter und anderer sozial benachteiligter Gruppen. In dieser Positionsbestimmung sind demzufolge weitreichende Konsequenzen hinsichtlich des Verhaltens der Partei zur Regierung, zur Christdemokratie, zu den Gewerkschaften und der Studentenbewegung enthalten.

18 Ein Abschnitt zur Sanierung der Industrie und zur Reform der Staatsunternehmen muß aus Platzgrïnden entfallen.

19 entfällt.

20 Die mit Korruption gepaarte Unfähigkeit wird zum Beispiel bei der Beseitigung der Erdbebenschäden đeutlich. Im Belicetal (Sizilien) sind mehrere Jahre nach dem Beben noch immer keine neuen Wohnbauten errichtet und die Bevölkerung muß immer noch in Behelfsunterkünften wohnen. Bei der Verteilung der Mittel, um die Schäden des Bebens in Friaul $1976 \mathrm{zu}$ beseitigen, soll der Parteiapparat der KPI sehr viel effizienter gearbeitet haben als die sehr viel umfangreichere staatliche Bürokratie. 
Damit also die linken Gemeinden und Regionen mit ihrer alternativen Politik bei wichtigen Maßnahmen und Projekten nicht zur Wirkungslosigkeit verdammt werden, macht die KPI die Forderungen nach einer breiten Dezentralisierung der Verwaltung und nach Autonomisierung lokaler und regionaler Institutionen sowie fast sämtlicher Bereiche des öffentlichen Sektors gegenüber Rom zu einem Kernpunkt der Reformprogrammatik.

Dezentralisierung und Autonomisierung zielen auf Ausweitung der demokratischen Kontrolle vor Ort hin. Ansätze dieser Reformprogrammatik sind bereits in den ,roten ${ }^{66}$ Gemeinden und Regionen realisiert worden. An diesen Ansätzen wird deutlich, was unter Dezentralisierung und Demokratisierung verstanden wird. Zunächst geht es um eine Vereinfachung der Bürokratie, um die Entscheidungen der Verwaltung tranparent $\mathrm{zu}$ machen undKorruption zu vermeiden. Weitergehend soll die Exekutive auf ihren ausführenden Charakter und auf koordinierende Tätigkeit zurïkgeführt werden. Dazu soll die demokratische Kontrolle der Exekutive durch gewählte Gremien vom Parlament bis zu den direkt gewählten Stadtteilräten, die vor allem die politischen Entscheidungen und Maßnahmen für ihren Lebensbereich treffen, ausgeweitet werden. Zu diesem Zweck muß aber die direkte Beteiligung der verschiedenen sozialen Gruppen an der Lösung der sie betreffenden Probleme gefördert und diese in einem breiten Lernprozeß zur Selbstverwaltung befähigt werden; es muß dementsprechend eine Vermittlung zwischen sozialen Bewegungen und Institutionen geschaffen werden, wie sie in den Stadtteilräten ansatzweise verwirlicht ist. Im Sozialfürsorgebereich sollen vor allem eine Entghettoisierung stattfinden und Formen der Selbstverwaltung erarbeitet werden.

In den roten Gemeinden und Regionen gibt es bereits eine Fülle von Erfahrungen bei der Realisierung dieser Reformprogrammatik von Dezentralisierung und Demokratisierung. Doch diese Ansätze, die gegen die Zentralisierung erkämpft worden sind, sind in der Krise außerordentlich gefährdet. Unter Ausnutzung der Krisenbedingungen sind in den letzten Monaten die Finanzen derartig verknappt worden, daß politische Maßnahmen, wie sie etwa die Stadtverwaltung von Bologna getroffen hatte, innerhalb der Schulreform, der Stadtplanung, oder innerhalb des öffentlichen Verkehrs (Nulltarif) zurückgedrängt oder gar unwirksam gemacht werden konnten. Die Reformprogrammatik für den öffentlichen Sektor verlangt vor allem in der Krise ein erhebliches Durchsetzungsvermögen der Arbeiterbewegung, zumal sie frontal auf die Bastionen einiger potenter DC-Gruppierungen stößt. Allerdings gibt es auch in der DC Gegentendenzen, und die rühren aus der für jedermann offenkundigen parasitären Unwirksamkeit der öffentlichen Verwaltung her. Teilweise bröckelt es im Einflußbereich der DC selber, und es sind Kräfte entstanden, die sich hinter die Reformprogrammatik stellen und sie unterstützen. Bemerkenswerterweise befüworten große Teile der staatlichen Polizei eine Gewerkschaft, die organisatorischer Teil der drei Bünde (CGIL-CISL-UIL) sein soll. Sie haben sich damit gegen eine autonome gelbe Gewerkschaft der Polizei ausgesprochen. Verbunden ist diese Entscheidung mit der Forderung nach einer Reform der staatlichen Polizei, die vor allem in Dezentralisierung und Entmilitarisierung bestehen soll. Zwar versucht die Andreotti-Regierung diese Entwicklung gesetzlich abzublocken, doch gibt es auch innerhalb der DC Unterstüt- 
zung für die Polizeireform, wie sie von den Linksparteien angestrebt wird. Mit einem entsprechenden Reformgesetz wäre allerdings noch keine Demokratisierung der Polizei erreich $t$, da vor allem die reale Durchsetzung erst in Gang gesetzt werden muß. Aber die Gefahr der Manipulierbarkeit der Polizei durch autoritäre oder neofaschistische Kräfte ist zumindest verringert.

\section{Die Gewerkschaften}

Die politischen Folgen der Zersetzungserscheinungen in der Krise haben auch die Gewerkschaftsbewegung ergriffen und bedrohen vor allem ihre für das Durchsetzungsvermögen der Arbeiterbewegung unerläßliche Einheit. Außer gegen die gleitende Lohnskala richtete sich die offensive Kampagne der Unternehmerverbände und der Regierung mit internationaler Schützenhilfe in den letzten Monaten gegen die entscheidende Machtposition der Gewerkschaften, gegen ihre Tarifautonomie in Form der differenzierten und betriebsnahen Tarifpolitik, also gegen das Recht der verschiedenen Gewerkschaftsinstanzen, jederzeit alle Aspekte des Arbeitsverhältnisses auf allen Ebenen zu verhandeln. Der Internationale Währungsfonds (IWF) forderte die Abschaffung dieser beiden entscheidenden Machtpositionen der Gewerkschaften als Bedingung für den von Italien gewünschten Kredit in Höhe von 530 Mio Dollar. Die Andreotti-Regierung hatte bereits einen entsprechenden Gesetzentwurf zur Reduzierung der Gewerkschaftsmacht vorbereitet. Die Gewerkschaften mußten ihre ganzen Kräfte auf die Verteidigung ihrer Positionen konzentrieren, um es nicht mit ihrem Finverständnis zu substantiellen Lohnsenkungen kommen und um nicht ihre Aktionsfreiheit gesetzlich einschränken zu lassen. Die Aufgabe der gleitenden Lohnskala und der betriebsnahen differenzierten Tarifpolitik hätte zur Unterminierung der Legitimationsbasis der Gewerkschaften und zur gefährlichen Spaltung der Lohnabhängigen in den Gewerkschaften geführt. Das war zweifellos eine wesentliche Zielsetzung der IWF-Bedingungen. Mit Hilfe der beiden Parteien KPI und PSI auf parlamentarischer Ebene konnten die Gewerkschaften ihre Stellungen einigermaßen verteidigen. So konnte der Gesetzentwurf zur Einschränkung der Tarifautonomie abgewehrt werden. Bei der gleitenden Lohnskala aber mußten die Gewerkschaften Zugeständnisse machen: aus der Bewegung der gleitenden Lohnskala sind die Preiserhöhungen der Transporttarife, der Elektrizität und der Zeitungen ausgenommen, was vermutlich zu schnellen Preiserhöhungen in diesen Bereichen führen und damit das vorgegebene Ziel hintertrieben wird, die Inflation durch Abschaffung der gleitenden Lohnskala aufzuhalten. Das wirkliche Ziel ist deutlich: mit den Gewerkschaften soll vor allem die vorhandene Stärke der Arbeiterbewegung getroffen werden.

Dieser zentrale Angriff auf wesentliche Machtpositionen der Gewerkschaften wurde vor allem auf zentraler Ebene abgewehrt. Dabei waren die dezentralen Gewerkschaftsinstanzen eher passive "Nachhut" der zentralen Verhandlungsgremien. Eben dieser Mangel an demokratischer Vermittlung und Übereinkunft hat zu Einbrüchen in den Gewerkschaften geführt und die notwendige Einheit gefährdet. Die Kritik an dem Abkommen zur Einschränkung der ,scala mobile' richtet sich auch gegen das 
Zustandekommen des Abkommens ohne die breite demokratische Beteiligung der dezentralen Gewerkschaftsinstanzen und der Masse der Lohnabhängigen. In der Tat sind die Zugeständnisse in dem Abkommen, gemessen an dem Druck, der auf den Gewerkschaften lastete, vergleichsweise gering (21). Als Hauptaufgabe stellt sich bei der zunehmenden Disgregation, die nun auch die Gewerkschaften selber ergreift, die Einheit innerhalb der Gewerkschaft wiederherzustellen und dabei auch die anderen sozialen Bewegungen wie die Studenten-, die Frauen- und die Arbeitslosenbewegung miteinzubinden.

Diese Einheit aber kann nach dem Selbstverständnis der Gewerkschaften vor allem im Kampf für einen alternativen Wirtschaftsprozeß und d. h. gegen die Arbeitslosigkeit, für neue Arbeitsplätze hergestellt werden.

In dieser Hinsicht haben die Gewerkschaften auf Konzernebene bereits zahlreiche Erfolge zu verzeichnen, was z. B. Investitionsverpflichtungen der wichtigsten Unternehmen im Süden zu der dort notwendigen Industrialisierung angeht (22). Doch sind diese tarifvertraglich vereinbarten Investitionsvorhaben fast allesamt papierene Zusagen geblieben und haben das Licht der Verwirklichung nicht erblickt. Diese Fülle von vertraglichen Einzelerfolgen produziert aber in ihrer Wirkungslosigkeit unter Umständen ein resignatives Bewußtsein über das gewerkschaftliche Durchsetzungsvermögen und befördert wiederum korporativistische Disgregationserscheinungen. Um dem entgegenzuwirken, müssen die Gewerkschaften ihre gesamte organisatorische Kraft auf die Durchsetzung der vertraglichen Vereinbarungen konzentrieren, um durch konkrete Erfolge die Kämpfe der Lohnabhängigen mit den Interessen der Arbeitslosen zu verbinden und so die Einheit der Arbeiterklasse zu erhalten oder herzustellen. Sie begegnen damit auch der Kritik von Wirtschaftsexperten der KPI und Amendolas, die den Gewerkschaften vorwerfen, sie hätten ausschließlich die Lebensbedingungen der beschäftigten Lohnabhängigen verteidigt und dabei die Probleme der Inflation und der Arbeitslosigkeit vernachlässigt. In diesem Zusammenhang drehen sich einigermaßen heftige Auseinandersetzungen um die Rolle der Gewerkschaften bei dem Kampf um neue Arbeitsplätze und eine alternative Wirtschaftspolitik. Amendola und die Wirtschaftsexperten der KPI gehen davon aus, daß sich die Gewerkschaften aktiv für die Senkung der Inflationsrate einzusetzen haben, daß mithin der Kampf für die gleitende Lohnskala, der Kampf zur Erhaltung der bestehenden Ar-

21 Bei einer Reihe yon zentralen und dezentralen Delegiertenversammlungen ist vor allem die mangelnde Vermittlung von zen traler Verhandlungsführung und betrieblichen Kämpfen - in vielen Betrieben wurden spontane Streiks durchgeführt - kritisiert worden. Allerdings muß dabei gesehen werden, daß yon einer Verselbständigung der Spitzen keineswegs die Rede sein kann. In den letzten Monaten sind mehrere regionale Generalstreiks organisiert worden, um die Geschlossenheit der Arbeiterklasse bei der Verteidigung ihrer Errungen schaften zu demonstrieren.

22 In betrieblichen Tarifverträgen werden nicht nur die Löhne ausgehandelt, sondern auch Investitionen zur Schaffung von Arbeitsplätzen minutiös festgelegt. Diese Verträge bleiben allerdings Papier, solange die Unternehmen sich in der Krise darauf zurückziehen können, Investitionen aus ökonomischen und finanziellen Gründen überhaupt zu reduzieren. 
beitsplätze und teilweise auch der gewerkschaftliche Anspruch auf bedingungslose Tarifautonomie in gruppenegoistische Tendenzen münden könnte. Sie fordern die Gewerkschaften zur Bereitschaft auf, sich verantwortlich in eine gesamtwirtschaftliche Wirtschaftsplanung einzubinden (23), deren Protagonisten die politischen Parteien sind. Dieser Aufforderung Amendolas liegt ein Begriff von Allgemeinwohl der Nation zugrunde, den die Gewerkschaften mit ihrer Tradition als Klassenorganisation nicht teilen können. Mit ihrem Verständnis des Allgemeinwohls, das sich grundsätzlich auf die Lage der Klasse der Lohnabhängigen bezieht und dabei diese nicht als eine besondere soziale Gruppe unter vielen begreift, können sich đie Gewerkschaften nicht erstrangig als Vorkämpfer gegen die Inflation erklären, die sie als vom Kapital produzierte begreifen, sondern müssen den Kampf für neue Arbeitsplätze mit der Verteidigung der bestehenden Arbeitsplätze und der Lebensbedingungen der Lohnabhängigen verbinden. Außerdem fürchten sie, daß bei einer Einbindung in eine Wirtschaftsplanung ihre Tarifautonomie geschwächt werden könnte und die demokratische Kontrolle ebenso wie die Möglichkeit, Druck auszubuien, erschwert werden könnte. Die Gewerkschaften wollen in ihrer Mehrheit direkt und über Massenkämpfe Einfluß auf die Wirtschaftsplanung ausüben und nicht in ihrer Aktionsfreiheit eingeschränkt werden. Die Gewerkschaften müssen daher die volle Autonomie zurïckgewinnen und verteidigen, da sie nur auf diese Weise das Durchsetzungsvermögen entwickeln können, das zur Realisierung der weitgehenden Reformkonzepte notwendig ist. Das wird umso dringlicher, falls der verstärkte Druck von KPI, PSI und auch PRI auf die DC dazu führt, daß eine breite Notstandsregierung doch noch zustande kommt; denn eine solche Regierung könnte zur Bindung der Gewerkschaften und damit zu einer Schwächung der Arbeiterbewegung führen.

Daher müssen die Gewerkschaften ihre zurückgedrängte Funktion, die allgemeine Vertretung der Lebens- und Arbeitsinteressen der Lohnabhängigen zu ïbernehmen, wiedergewinnen. Auch innerhalb der KPI stehen sich unterschiedliche lang. fristige politische Gesellschaftskonzeptionen von Rolle der Parteien und der Gewerkschaften gegenüber: Sind die Gewerkschaften mit ihrer Rätestruktur die grundlegenden Organisationen der Arbeiterbewegung, die damit auch die Urform des späteren Arbeiterstaats darstellen (24) und nehmen die Parteien dabei nur eine pluralistische Vermittlungsfunktion zwischen sozialen Bewegungen und Institutionen ein oder sind die Gewerkschaften auf die vorwiegend ökonomischen Interessen der Lohnabhängigen konzentriert und überlassen das Feld der politischen und sozialen Organisation vornehmlich den Parteien? Die Krise hat auch diese Alternative deutlich gemacht angesichts der Frage, welche Rolle die Gewerkschaften unmittelbar in der Krise einnehmen sollen. Dennoch ist die organisatorische Einheit dadurch nicht bedroht; dazu ist die eingeubte Praxis der offenen, öfentlichen, solidarischen aber heftigen Auseinandersetzungen innerhalb der italienischen Arbeiterbewegung zu stark verankert; wohl aber ist die Aktionsfähigkeit der Gewerkschaften und der KPI angesichts der

23 So jüngst auch der Ökonomi Luigi Spaventa in: Rinanscita Nr. 14, 8.4.77, S. 9

24 Eine runktionsbestimmung der Geverkschaften in dieser Weise hat bereits Gramsci vorgenommen. Bruno Trentin hat erst jüngst diese Vorstellung weiterentwicklet. Vgl. Bruno Trentin, Da sfruttati a produttori, Bari 1977 
drängenden Probleme eingeschränkt.

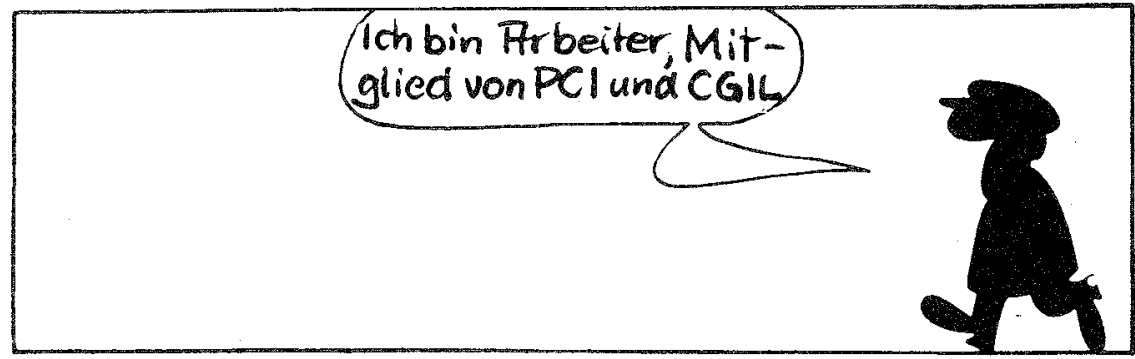

$\mathrm{HIS} \mathrm{PCl}$-Mitglied unierstütze idn die Fnareotti-Reg.
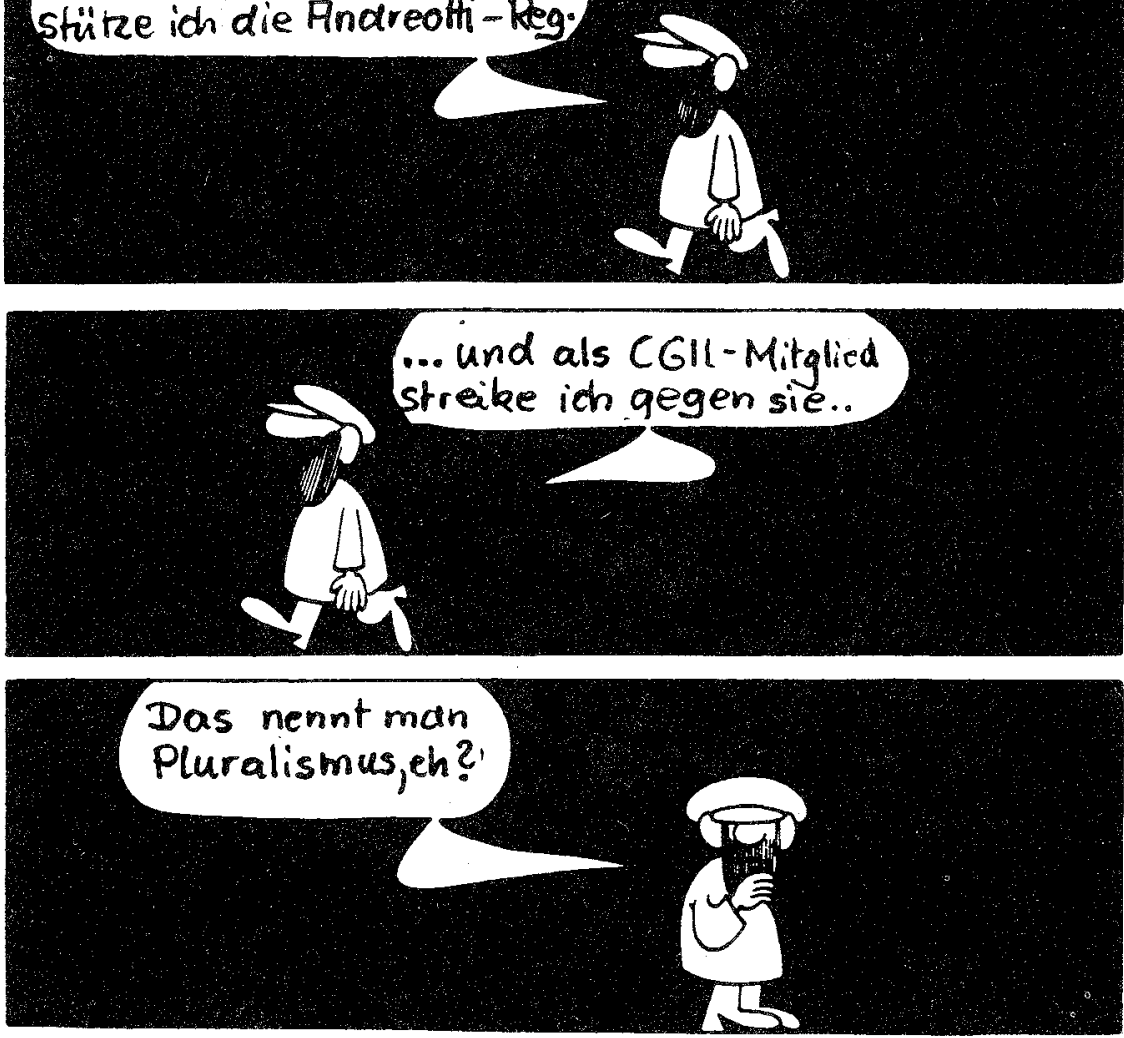

Zeichnung: A. Chiappori (Panorama vom 7. Dez. 1976) 


\section{4. „Eurokommunismus" als Antwort auf die Wirkungen der Krise?}

Die italienische Arbeiterbewegung steht in der gegenwärtigen Krise vor Aufgaben, die bislang noch von keiner proletarischen Partei positiv gelöst worden sind: nämlich in der Krise Mitte der 70er Jahre anders als in den 30er Jahren von den autoritären, faschistischen Tendenzen nicht überrollt zu werden, sondern Machtpositionen zu erobern, die gesellschaftliche Hegemonie der Arbeiterklasse auszuweiten, ein Stück weiter auf dem Weg zum Sozialismus voranzukommen. Die deutsche Arbeiterbewegung - ähnlich wie die italienische 1923 - ist 1933 gescheitert, nicht weil sie ,objektiv" zu schwach war, um die faschistische Machtergreifung zu verhindern, sondern weil sie ihre eigene Zerrissenheit nicht überwinden konnte, ja sie zum Teil noch beispielsweise mit der Sozialfaschismus-These der KPD oder mit dem wütenden Antikommunismus von Teilen der SPD - kultivierte. Die Disgregation der Arbeiterbewegung zu verhindern, stellt sich folglich als entscheidende Aufgabe in der gegenwärtigen Krise. Wenn dies nicht gelingt, dann ist ein Rückschlag wie in den 30er Jahren mit entsprechend katastrophalen Konsequenzen für die Völker im Bereich des Mög. lichen.

In diesem Problemfeld ist der sogenannte „Eurokommunismus“ (25) angesiedelt: als eine mögliche strategische Antwort auf die Wirkungen der Krise. Dabei ist es weniger bedeutsam, daß Eurokommunismus die Abkehr vom Moskauer Zentrum impliziert und folglich den kommunistischen Parteien des Westens die Möglichkeit eröffnet, ohne Berücksichtigung der Interessen des ,Weltkommunismus" ihre nationalen Kompromisse mit den jeweiligen nationalen Bourgeoisien zu schließen (26); unter diesem Aspekt ist Eurokommunismus nichts anderes als die Fortsetzung der „polyzentristischen“ Tendenzen in der kommunistischen Weltbewegung seit dem Konflikt mit Tito Ende der 40er Jahre. Uns scheint ein anderer Aspekt des sogenannten Eurokommunismus wichtiger zu sein, nämlich der darin implizierte Versuch, eine Strategie gegen die Gefahr der Disgregation nicht nur innerhalb der nationalen Arbeiterklassen sondern auch auf europäischer Ebene zu entwickeln.

Gerade angesichts von Tendenzen der bürgerlichen Klassen und Regierungen, in der Krise die „Steuerungskapazität" des kapitalistischen Staates dadurch zu vergrößern, daß die demokratischen Möglichkeiten der Einflußnahme auf staatliche Entscheidungen autoritär verringert werden, insbesondere die Organisationen der Arbeiterklasse zurückgedrängt werden, um die Krisenbereinigung durchführen zu können, ist eine Demokratisierungs- und Reformstrategie der Arbeiterbewegung von außerordentlicher Bedeutung. In diesem Rahmen sind die Ansätze vor allem der fránzösischen, spanischen und italienischen kommunistischen $P$ arteien zu sehen, sich als Garanten demokratischer Entwicklungen gegen autoritäre Tendenzen, für einen gesellschaftiichen Pluralismus als Basis von Hegemonie der Arbeiterbewegung, für Strukturrefor-

25 Der Begriff ist ursprïnglich eine Schöpfung der bürgerlichen Presse, aber von den süheuropäischen kommunistischen Parteien positiv aufgegriffen worden.

26 Vgl dazudie austihnliche Analyse des Cesim (Centro studi internazionali maristi), C'e an-

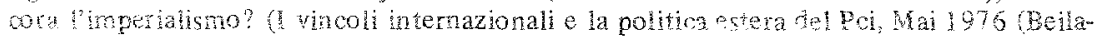
gin zolia Continua, N. 137 ) 
men zu profilieren. Damit wird ansatzweise versucht, in einer Revolutionsstrategie die besonderen Verhältnisse in den kapitalistischen Industrieländern zu berïcksichtigen, sowohl hinsichtlich der Klassenstruktur als auch hinsichtlich der Erfahrungen der Arbeiterklasse aus den Kämpfen der vergangenen 10 Jahre.

In der gegenwärtigen Krise stellt es sich sehr klar heraus, welche große Gefahr für die westeuropäische Arbeiterbewegung die mittlerweile traditionelle Spaltung in die kommunistische und die sozialdemokratische Bewegung bedeuten kann. Denn die darin zum Ausdruck kommende Disgregation wird gegenwärtig gerade von der Bourgeoisie international benutzt, um die entwickelten Klassenbewegung zurückzudrängèn. Wenn Italien zur Überbrückung der Zahlungsbilanzkrise einen Kredit von der EG oder vom IWF haben will, dann werden von diesen internationalen Institu tionen des Kapitals Auflagen durchzusetzen versucht, die eindeutig die Schwächung der italienischen Gewerkschaften und Linksparteien zum Ziel haben. Die Führung der deutschen Sozialdemokratie spielt bei diesen Manövern, die nur die konsequente Fortsetzung der Stützungsaktionen bürgerlicher Kräfte in Portugal oder in Spanien darstellen, eine hervorragende Rolle. Die Einbindung der westdeutschen Arbeiterklasse in die sozialdemokratische Regierungspolitik, auch die Bindung der Gewerkschaften in der BRD an diese Politik und deren Fortsetzung auf internationaler Gewerkschaftse bene, kann somit zu einer unmittelbaren Schwächung der Arbeiterbewegungen Südeuropas führen, zumindest weil der Widerstand gegen die Versuche des Kapitals zur Krisenbewältigung auf Kosten der Arbeiterklasse in der kapitalistischen Hegemonialmacht Westeuropas de facto zusammengebrochen ist. Die Erkenntnis, daß der Attacke des Kapitals auf die Arbeiterbewegung in der Krise nur gemeinsam im westeuropäischen Bereich begegnet werden kann, ist daher einer der wichtigsten Aspekte des „Eurokommunismus“ (27). Daß die Herstellung dieser Gemeinsamkeit, d. h. aber gerade die Überwindung der Disgregation in der Arbeiterbewegung auf internationaler Ebene, auch strategische Neuformulierungen gegenüber den traditionellen Konzeptionen der Arbeiterbewegung erforderlich macht, dürfte einsichtig sein.

Unter diesem Gesichtspunkt betrachtet ist die Politik der westeuropäischen Gewerkschaften und der Parteien der Arbeiterbewegung in Westeuropa für unsere Überlegungen in der BRD von Bedeutung. Es geht dabei nicht darum, ,Eurokommunismus“ als „Modell“ übertragen zu wollen (28), oder angesichts der Misere der westdeutschen Arbeiter bewegung nach den aus den Arbeiterkämpfen in Italien 1969 hervorgegangenen Gruppen der neuen Linken ein neues Identifikationsobjekt zu suchen, sondern darum, die Aufgaben der westdeutschen Linken im historischen $\mathrm{Zu}$ -

27 So bestimmt Berlinguer im Vorwort zu Enrico Berlinguer, La politica internazionale dei oomunisti italiani, Roma 1976 die Funktion des Eurokommunismus. Vgl. auch Corriere della Sera, 24.10.1976

28 In dieser Hinsicht ist die Konsequenz von Detlev Claussen, Heinrich Grun und David Wit\{enberg, Italien - Der diskrete Charme des Eurokommunismus?, in: links Nr. 87, April 1977 fehlgehend. Aus der richtigen Kritik an bloßen Modellüberlegungen oder Indentifikationen mit politischen Gruppen in anderen Ländern kann natürlich nicht gefolgert wer* den, daß aus den Erfahrungen von Klassenorganisationen - Gewerkschaften, sozialistische und kommunistische Gruppen und Parteien - nicht mehr für unsere eigene politische Praxis gelernt werden könne. 
sammenhang der westeuropäischen Entwicklung zu reflektieren und - zum Beispiel - aus den Schwierigkeiten der italienischen Arbeiterbewegung und insbesondere der KPI, und aus den Ansatzpunkten, sie zu überwinden, Lehren für unsere eigene politische Prax is zu ziehen.

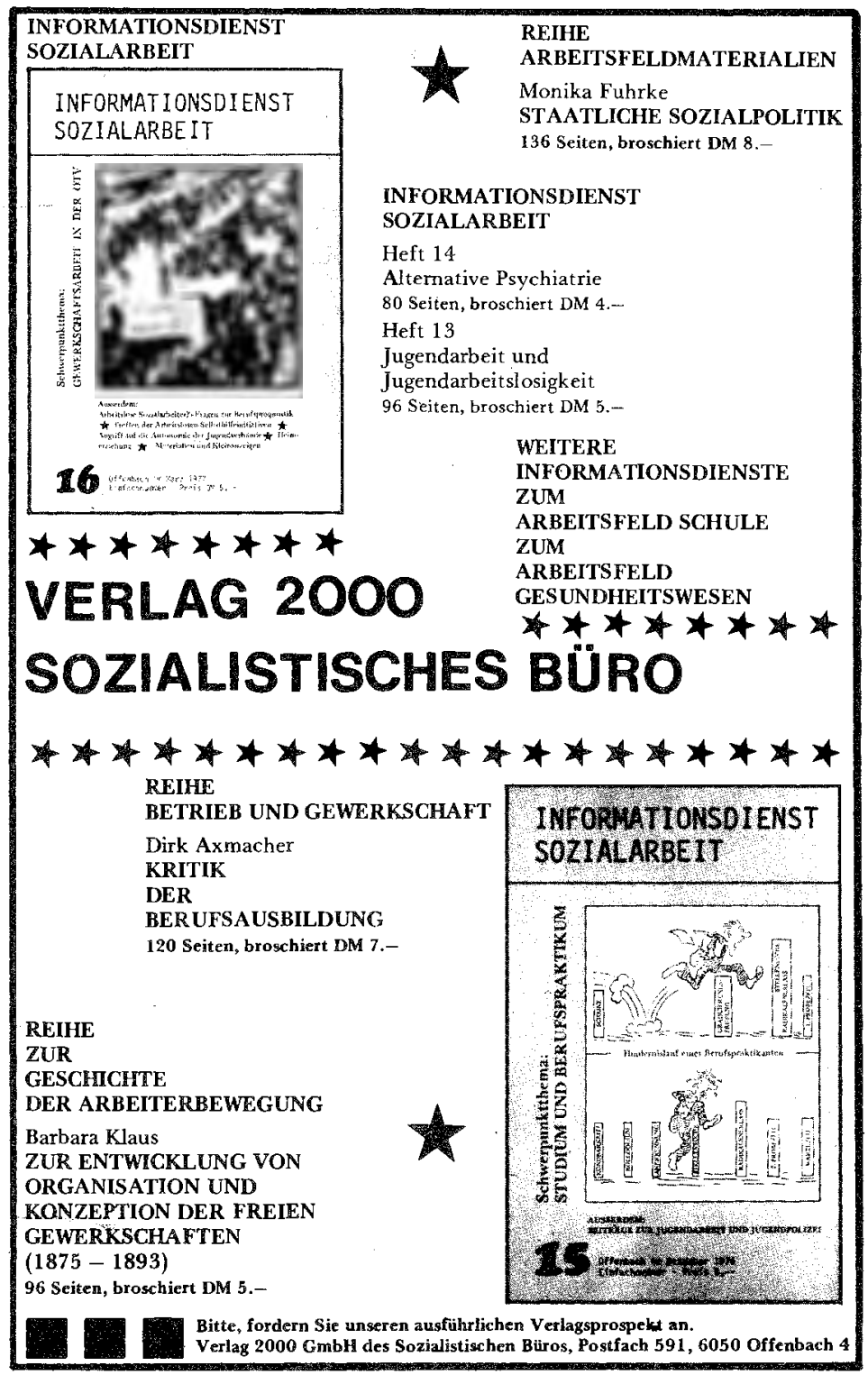

